Original

\title{
Adaptive underfrequency load shedding using particle swarm optimization algorithm
}

\author{
Abbas Ketabi*, Masoud Hajiakbari Fini \\ Department of Electrical Engineering, University of Kashan, Kashan, Iran \\ Received 9 September 2015; accepted 9 December 2016 \\ Available online 16 February 2017
}

\begin{abstract}
Underfrequency load shedding plays an important role in prevention of the power system blackout. The common load shedding methods are based on measuring the frequency first derivative; therefore, an error in measurement process can highly affect their performance. Also, for proper performance of these load shedding schemes, the exact value of some parameters of power system is needed. Any error in estimation of these parameters reduces the reliability of these load shedding schemes. In this paper, an underfrequency load shedding method based on the forecast minimum frequency of system is proposed. In this method, the samples of the power system frequency are taken after disturbance; then, particle swarm optimization algorithm is used to forecast the minimum frequency based on these samples. To verify the effectiveness of the proposed load shedding scheme, its performance has been compared with a newly suggested method.

(C) 2017 Universidad Nacional Autónoma de México, Centro de Ciencias Aplicadas y Desarrollo Tecnológico. This is an open access article under the CC BY-NC-ND license (http://creativecommons.org/licenses/by-nc-nd/4.0/).
\end{abstract}

Keywords: Blackout; Minimum frequency; Power deficit; Underfrequency load shedding

\section{Introduction}

Maintaining the power system frequency within the permissible limits is an important control task which in normal conditions is carried out by means of load frequency control (Hajiakbari Fini, Yousefi, \& Alhelou, 2016; Ketabi \& Hajiakbari Fini, 2015a; Kundur, 1994). But, when a sudden power deficit occurs as a result of outage of large generating units or islanding of some parts of power system, even if the existing generating units have enough spinning reserve to supply the demand, their response is not rapid enough to stop the frequency excursion and prevent the operation of generating units protective relays. This may lead to outage of some of the generating units. Consequently, outage of a generating unit can worsen the situation and decrease the frequency to a lower level; therefore, the relays of other generating units might trip and lead to power system blackout. A solution which is widely used in power systems for overcoming this situation is the implementation of underfrequency load

\footnotetext{
* Corresponding author.

E-mail address: aketabi@kashanu.ac.ir (A. Ketabi).

Peer Review under the responsibility of Universidad Nacional Autónoma de México.
}

shedding (UFLS) schemes (Ketabi \& Hajiakbari Fini, 2015b; Laghari, Mokhlis, Bakar, \& Mohamad, 2013).

In literature, a number of strategies have been implemented for UFLS (Aik, 2006; Anderson \& Mirheydar, 1990; Anderson \& Mirheydar, 1992; Berdy, 1968; Chuvychin, Gurov, Venkata, \& Brown, 1996; Concordia, Fink, \& Poullikkas, 1995; Delfino, Massucco, Morini, Scalera, \& Silvestro, 2001; Halevi \& Kottick, 1993; Hong \& Chen, 2012; Hong \& Wei, 2010; Larsson, 2005; Lokay \& Burtnyk, 1968; Lopes \& Mitchell, 2000; Maliszewski, Dunlop, \& Wilson, 1971; Pahwa, Scoglio, Das, \& Schulz, 2013; Rudez \& Mihalic, 2011a, 2011b, 2011c, 2015; Terzija \& Koglin, 2002; Thalassinakis \& Dialynas, 2004). Conventional method is the first generation of UFLS scheme which has been introduced in (Lokay \& Burtnyk, 1968) and developed by some other authors (Aik, 2006; Berdy, 1968; Concordia et al., 1995; Delfino et al., 2001; Halevi \& Kottick, 1993; Maliszewski et al., 1971; Thalassinakis \& Dialynas, 2004). The first stage in this method is determination of the worst probable generation loss event which results in the highest initial rate of frequency decay. Then, the amount of load shedding which ensures that the frequency will not deviate below the minimum permissible value should be specified. After that, the number and size of load shedding steps are determined based on trial and error to achieve an acceptable 
operation in case of the worst probable contingency. Determining the worst probable contingency and also the size and number of load shedding steps are not easy tasks. Also, in this method a specified amount of load is shed in every stage without considering the amount of power deficit. Hence, it is probable that overshedding occurs and the frequency increases above the maximum permissible value.

A number of algorithms have been used to optimize the conventional UFLS method (Aik, 2006; Halevi \& Kottick, 1993; Hong \& Chen, 2012; Hong \& Wei, 2010; Lopes \& Mitchell, 2000). In Hong and Chen (2012) a UFLS scheme is optimized based on genetic algorithm (GA). To maximize the lowest swing frequency and minimize the amount of shed load, GA is implemented in Hong and Wei (2010). The drawbacks of conventional UFLS can be remedied by using the adaptive UFLS method proposed in Anderson and Mirheydar (1992). In this method, using swing equation, the amount of power deficit is calculated based on the frequency derivative immediately after the disturbance. Therefore, there is no need to determine the worst probable contingency and an amount of load based on the calculated power deficit is shed at the specified stages. In Rudez and Mihalic (2011a, 2011b) the effect of load parameters on adaptive UFLS scheme is investigated. The adaptive UFLS methods estimate the power deficit using swing equation. Hence, these methods are dependent on the equivalent inertia constant of the power system and any change in this parameter may highly affect its performance. Moreover, the estimated power deficit is directly related to the frequency derivative at the moment the disturbance occurs. Therefore, errors in frequency derivative will degrade the reliability of this UFLS scheme.

In Rudez and Mihalic (2011c), a new UFLS scheme based on the minimum frequency of system after disturbance is proposed. As authors claimed, in this scheme no knowledge of power system parameters is needed. In this method, the frequency second derivative is estimated based on Newton method; then, the minimum frequency is forecasted using numerical integration. After that, the amount of load to be shed is determined based on the minimum frequency. However, measuring the frequency second derivative is a challenging problem.

In this paper, a load shedding scheme is proposed in which the frequency of system after occurrence of disturbance is forecasted using the particle swarm optimization (PSO) algorithm. Then, the minimum frequency of system is obtained as described in Section 2. After that, the amount of load which is necessary to be shed to maintain the frequency of system within the permissible limits is determined. Afterwards, the determined amount of the load can be shed in several steps as described in Section 3. In Section 4, requirements for implementing the proposed UFLS method in real power systems have been pointed out. In Section 5 , the proposed load shedding scheme is compared with a newly proposed UFLS method through simulation results.

\section{Implementing PSO for frequency estimation}

When a disturbance such as generating units outage occurs in a power system and causes a power deficit, frequency drops

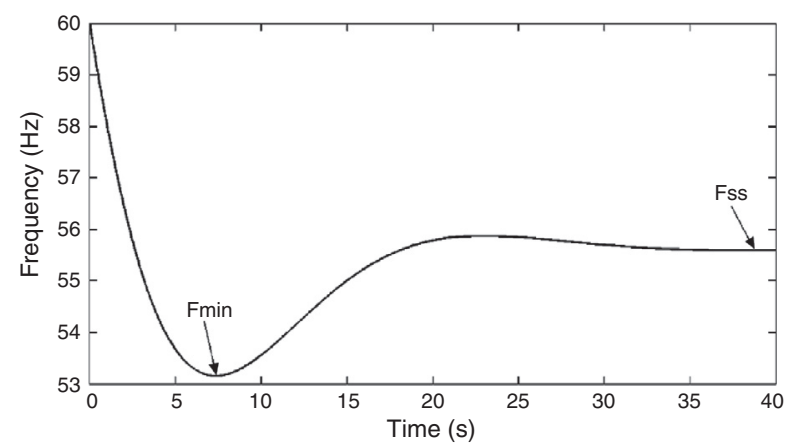

Fig. 1. Frequency drop after occurrence of power deficit in a typical power system.

as shown in Figure 1. This frequency drop can be represented by following equation (Anderson \& Mirheydar, 1990):

$\Delta \omega=\left(\frac{R \omega_{n}^{2}}{D R+K_{m}}\right)\left(\frac{\left(1+T_{R} s\right) P_{\text {step }}}{s\left(s^{2}+2 \xi \omega_{n} s+\omega_{n}^{2}\right)}\right)$

where

$\omega_{n}^{2}=\frac{D \times R+K_{m}}{2 H \times R \times T_{R}}$

$\zeta=\frac{2 H \times R+\left(D \times R+K_{m} \times F_{H}\right) \times T_{R}}{2\left(D \times R+K_{m}\right)} \times \omega_{n}$

The objective is to forecast the frequency deviation, as precisely and quickly as possible, using the minimum possible measured frequency samples. Hence, for curve fitting, a function should be chosen that represents the frequency of system precisely and has the minimum number of parameters to be estimated by PSO. By trial and error it is found that in the studied power system, the frequency deviation of system up to $3 \mathrm{~s}$ after the disturbance can be estimated accurately by fitting a cubic function on frequency deviation samples measured within $0.1 \mathrm{~s}$ after occurrence of the power deficit. So, the frequency deviation samples taken within $0.1 \mathrm{~s}$ after the beginning of the frequency decay is used by PSO algorithm to determine the parameters of the following cubic function:

$\Delta f(t)=a t+b t^{2}+c t^{3} \quad 0 \leq t \leq 3$

As it is shown in Figure 2, frequency deviation of the system up to $3 \mathrm{~s}$ after the beginning of the frequency decay is forecasted

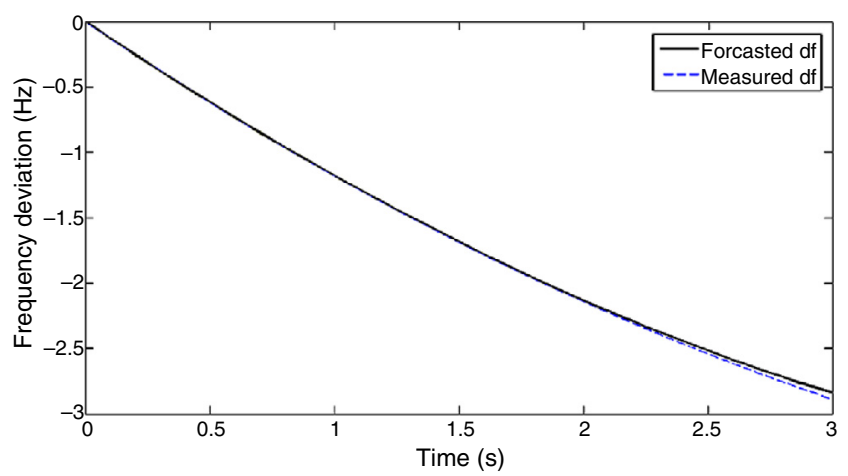

Fig. 2. Forecasted frequency deviation up to $3 \mathrm{~s}$ after disturbance with a cubic function. 


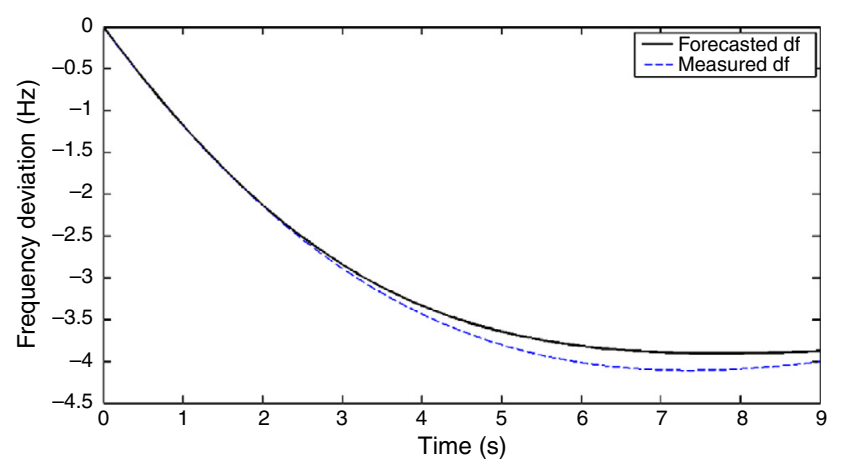

Fig. 3. Forecasted frequency deviation until reaching the minimum frequency.

with a high accuracy. Hence, this data can be used to estimate the frequency deviation of system until reaching the minimum frequency. For this purpose another curve fitting is required. It is found that the frequency deviation of system up to the point of minimum frequency can be estimated accurately by fitting the curve shown in (5) on the frequency deviation data obtained in previous step.

$\Delta f(t)=a+b e^{-c t} \sin (d t-e)$

Figure 3 shows that, using the proposed method, the frequency deviation is forecasted with a high precision until the point frequency reaches the minimum value.

To use PSO for obtaining the parameters of above mentioned functions $(\Delta f(t))$, it is necessary to determine a suitable objective function to be minimized. In this paper, the following series is selected as the objective function:

Cost $=\sum_{i=0}^{n} a b s\left(\Delta f_{\text {est }}(i)-\Delta f_{\text {meas }}(i)\right)$

where $\Delta f(i)_{\text {meas }}$ and $\Delta f(i)_{e s t}$ are, respectively, the $i$ th sample of measured and estimated frequency deviation and $n$ is the number of frequency deviation samples.

The PSO parameters which are used in this work are given in Table 1. Based on analysis of frequency response of the studied power system, we found that the parameters of (4) and (5) are located between -5 and 5 . Hence, -5 and 5 are assigned to $x_{\min }$ and $x_{\text {max }}$, respectively. For $c_{1}, c_{2}, w, w_{\text {damp }}$ and $v_{\max }$, typical values usually used in solving engineering problems by PSO have been chosen. Max-iteration and $N_{\text {pop }}$ have been selected based on trial and error to achieve satisfactory results.

Table 1

Parameters of PSO used for fitting the cubic and main function.

\begin{tabular}{lll}
\hline Parameters & Cubic function & Main function \\
\hline$c_{1}$ and $c_{2}$ & 2 & 2 \\
$x_{\min }$ & -5 & -5 \\
$x_{\max }$ & 5 & 5 \\
$d x$ & $x_{\max }-x_{\text {min }}$ & $x_{\max }-x_{\text {min }}$ \\
$w$ & 1 & 1 \\
$w_{\text {damp }}$ & 0.99 & 0.99 \\
$v_{\text {max }}$ & $0.1 \times d x$ & $0.1 \times d x$ \\
$M_{\text {Max }}$-iteration & 100 & 100 \\
$N_{\text {pop }}$ & 100 & 200 \\
\hline
\end{tabular}

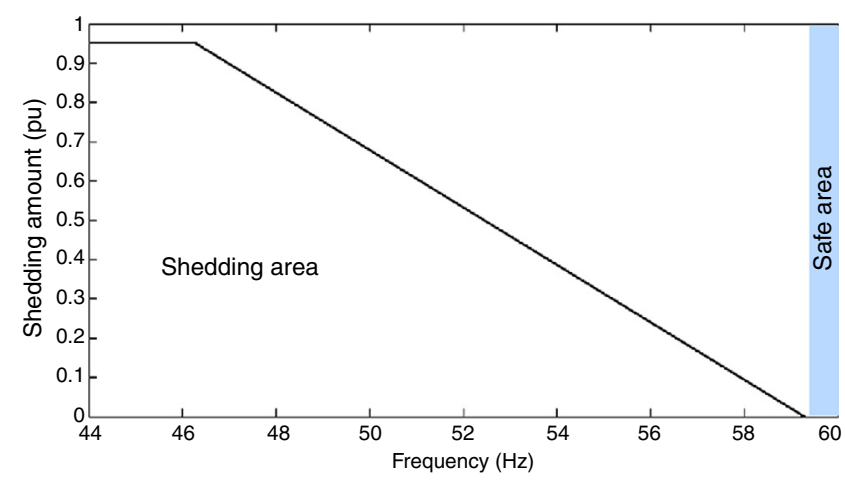

Fig. 4. Adopted load-shedding concept for a particular shedding step, based on the frequency forecast.

Details on PSO algorithm would be found in Eberhart and Kennedy (1995). Also, Pseudo Code for PSO is presented in Van Den Bergh and Engelbrecht (2001).

\section{Using the forecasted minimum frequency for load shedding}

In the previous section, it was shown that using the proposed method, the minimum frequency would be estimated precisely. In this section, it will be explained how to make use of the forecasted minimum frequency to determine the amount of load to be shed to prevent frequency excursion beyond the permissible limits.

The diagram in Figure 4 shows the relation between the forecasted minimum frequency and the amount of load which is necessary to be shed. In fact, this curve can be implemented to determine the proper amount of load shedding using the forecasted minimum frequency. Then, load shedding is carried out in three or four steps depending on the value of power deficit and after every step, the value of power deficit is estimated for the next step of load shedding using the procedure explained in the previous section. The load shedding process can be summarized as follows:

In the first step of load shedding, an amount of load equal to $30 \%$ of forecasted power deficit is shed. After that, the frequency of system until $0.1 \mathrm{~s}$ after load shedding is sampled again. Then, the minimum frequency; and therefore; the amount of power deficit are forecasted again. Afterwards, in the second step, an amount of load equal to $30 \%$ of forecasted power deficit is shed.

For the third step, if the amount of forecasted power deficit is less than 0.2 P.U., the amount of load shedding will be equal to forecasted power deficit. Otherwise, the shed load in the third step is equal to $50 \%$ of forecasted power deficit and the remained power deficit is compensated in the fourth step of load shedding. The flowchart of this load shedding scheme is shown in Figure 5. As simulation results show, for lower values of power deficit the minimum frequency is forecasted with higher accuracy. Therefore, the minimum forecasted frequency of system in every step will be more accurate in comparison with the previous step. So, the minimum frequency will be estimated precisely at the last step of load shedding and shedding the suitable amount of load is guaranteed. 


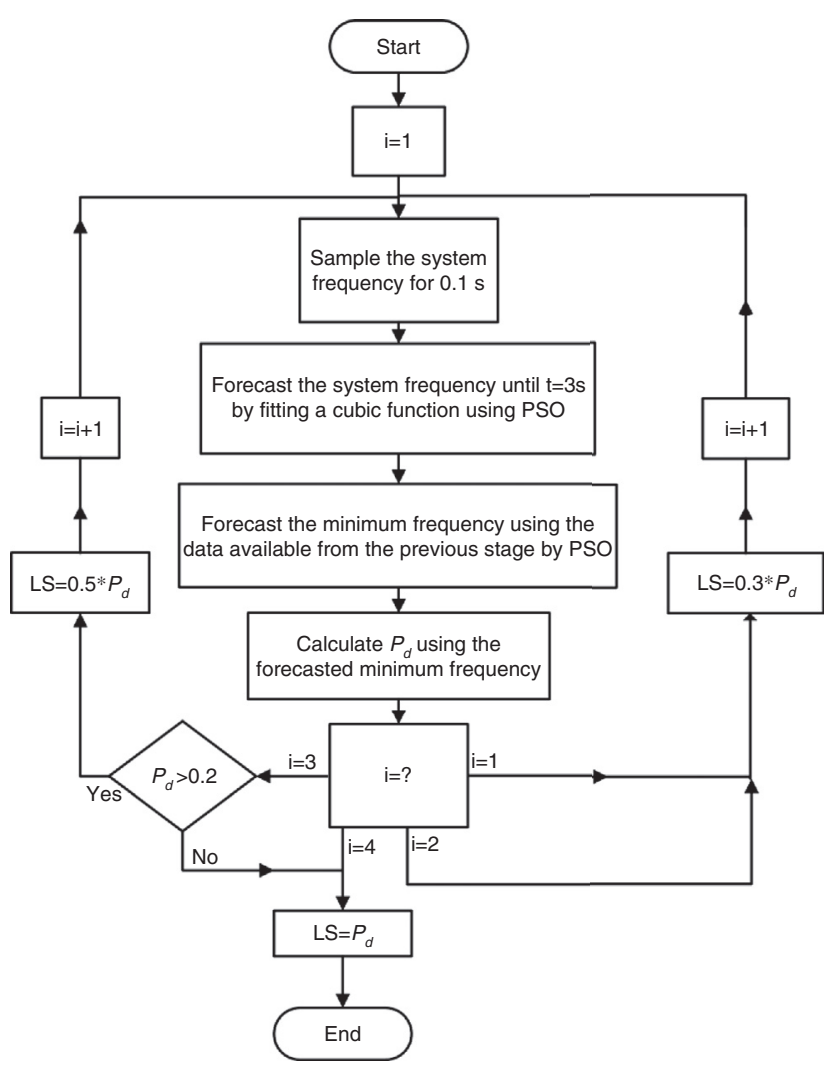

Fig. 5. The flowchart of proposed load shedding scheme.

\section{Requirements for implementing the proposed UFLS scheme in real power systems}

To keep the precision of the proposed UFLS method in real world power systems, frequency estimation should be carried out based on a measured frequency with the minimum oscillations. Center of inertia frequency, which is calculated as follows, would be a good candidate for this purpose:

$f_{C O I}=\frac{\sum_{i=1}^{n} H_{i} \cdot f_{i}}{\sum_{i=1}^{n} H_{i}}$

where $n$ is the number of generating units and, $f_{i}$ and $H_{i}$ are the frequency and inertia constant of the $i$ th generating unit.

Frequency of each generating unit would be measured using phasor measurement units and transmitted to the control center to be used in the UFLS scheme. After determining the amount of load to be shed at each stage, based on the load priorities, trip signal is sent to the selected load shedding relays. It is worth mentioning that for transmitting the measured frequencies and trip signals, fast communication links such as optic fiber is required.

\section{Simulation results}

To carry out studies on frequency stability and load shedding schemes, different models of power system can be used. System frequency response (SFR) model is one of the models widely used for load frequency control and UFLS studies (Aik, 2006; Anderson \& Mirheydar, 1990; Chang-Chien, An, Lin, \& Lee, 2012; Ketabi \& Hajiakbari Fini, 2014, 2015a; Sigrist, Egido,
Table 2

The parameters of test system.

\begin{tabular}{llllll}
\hline$H$ & $D$ & $F_{H}$ & $T_{R}$ & $K_{m}$ & $R$ \\
\hline 7 & 0.5 & 0.28 & 8 & 0.951 & 0.15 \\
\hline
\end{tabular}

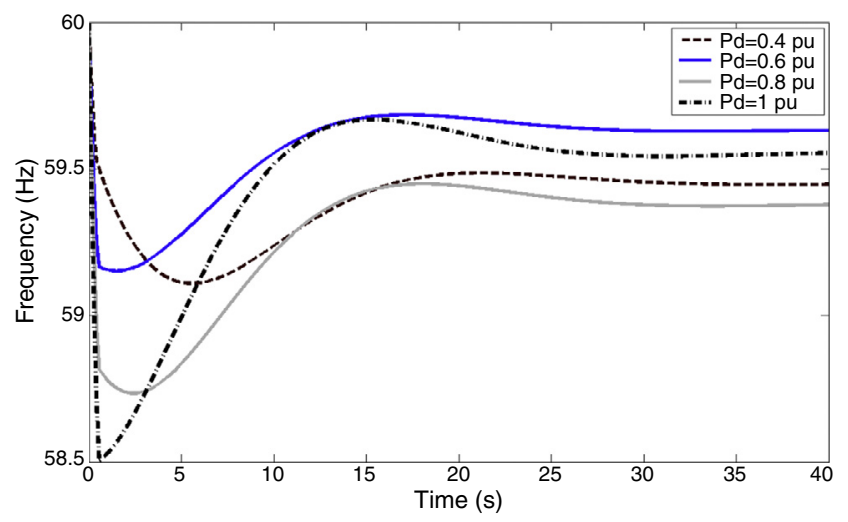

Fig. 6. The frequency of power system; load shedding scheme is activated due to different amount of power deficit.

Sanchez-Ubeda, \& Rouco, 2010). Due to its simplicity and accuracy, for simulation studies, we have used this model with the typical parameters given in Table 2 (Chang-Chien et al., 2012). Details regarding the SFR model have been presented in (Aik, 2006; Amjady \& Fallahi, 2010). It should be mentioned that the aim of the proposed load shedding scheme is to maintain the minimum frequency $\left(f_{\min }\right)$ and the steady state frequency $\left(f_{s s}\right)$ above 58.4 and $59.2 \mathrm{~Hz}$, respectively.

The proposed UFLS scheme is tested in case of power deficit ranged from 0.4 to 1 P.U. The results shown in Figure 6 certify that this UFLS scheme responses rapidly to the disturbance and maintains the frequency within permissible limits. For further clarifying, the detailed results of load shedding including the amount of shed load in every step, $f_{\min }$ and $f_{s s}$ are given in Table 3.

To show the effectiveness of the proposed load shedding method, it is compared with the adaptive load shedding method proposed in Rudez and Mihalic (2011a). For a thorough comparison, several case studies including the impact of power system parameters change on the schemes are carried out. In this paper the 'adaptive method' and 'novel method' refer to load shedding methods proposed in Rudez and Mihalic (2011a) and this paper, respectively.

\subsection{Case 1}

In this part, the performance of the two load shedding schemes in case of a power deficit equal to 0.8 P.U. is examined. It is clear from Figure 7 that, in this case, both load shedding schemes can maintain the frequency of system within the allowed limits. The detailed comparative results of both load shedding schemes are given in Table 4. 
Table 3

Detailed results of proposed load shedding scheme.

\begin{tabular}{|c|c|c|c|c|c|c|c|}
\hline Power deficit (P.U.) & Step 2 & Step 2 & Step3 & Step 4 & Total shed & $f_{\min }$ & $f_{s s}$ \\
\hline 0.4 & 0.1099 & 0.0836 & 0.1438 & - & 0.3373 & 59.11 & 59.45 \\
\hline 0.5 & 0.1245 & 0.1137 & 0.1235 & 0.0967 & 0.4584 & 59.27 & 59.63 \\
\hline 0.6 & 0.1370 & 0.1205 & 0.1751 & 0.1257 & 0.5583 & 59.19 & 59.63 \\
\hline 0.7 & 0.1510 & 0.1340 & 0.1749 & 0.1874 & 0.6473 & 58.96 & 59.53 \\
\hline 0.8 & 0.1677 & 0.1384 & 0.2064 & 0.2169 & 0.7294 & 58.73 & 59.38 \\
\hline 0.9 & 0.1850 & 0.1574 & 0.2255 & 0.2913 & 0.8592 & 58.66 & 59.64 \\
\hline 1 & 0.2022 & 0.1706 & 0.2587 & 0.3178 & 0.9493 & 58.5 & 59.55 \\
\hline
\end{tabular}

Table 4

Detailed result of novel and adaptive load shedding when the exact value of $H$ is known.

\begin{tabular}{|c|c|c|c|c|c|c|c|}
\hline Method & Step 1 & Step 2 & Step 3 & Step 4 & Total shed & $f_{\text {min }}$ & $f_{s s}$ \\
\hline Novel & 0.1677 & 0.1384 & 0.2064 & 0.2169 & 0.7294 & 58.73 & 59.38 \\
\hline Adaptive & 0.28 & 0.24 & 0.16 & 0.12 & 0.8 & 58.4 & 59.99 \\
\hline
\end{tabular}

\subsection{Case 2}

In adaptive method the power deficit is estimated using the following equation (Ketabi \& Hajiakbari Fini, 2015b; Rudez \& Mihalic, 2011a):

$P_{d}=\left.\frac{2 H}{60} \frac{d f}{d t}\right|_{t=0}$

where, $H$ is the inertia constant of the power system. In this method, the estimated power deficit is directly related to system inertia constant. Therefore, any difference between the value of $H$ used for estimating the power deficit and the real value of $H$ has a direct impact on the amount of calculated power deficit. So, if for any reason, for example an error in estimation of $\mathrm{H}$ or variations in inertia constant of system, there is a difference between the value of $H$ used in adaptive load shedding scheme and real value of $H$, the amount of calculated power deficit will be considerably different from the actual value. Consequently, this will result in malfunction of adaptive load shedding scheme.

In cases which the real $1 / H$ of power system is higher than the value used in the load shedding scheme, the estimated power deficit will be higher than the actual power deficit. But, due to the additional conditions which can cancel some steps of load shedding, the adaptive UFLS scheme will not result in overshedding. It is clear from Figure 8 that both load shedding schemes have

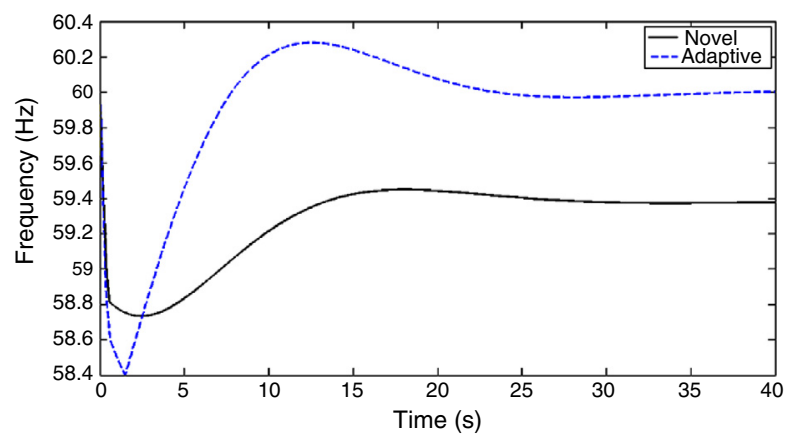

Fig. 7. Comparison of novel and adaptive load shedding schemes.

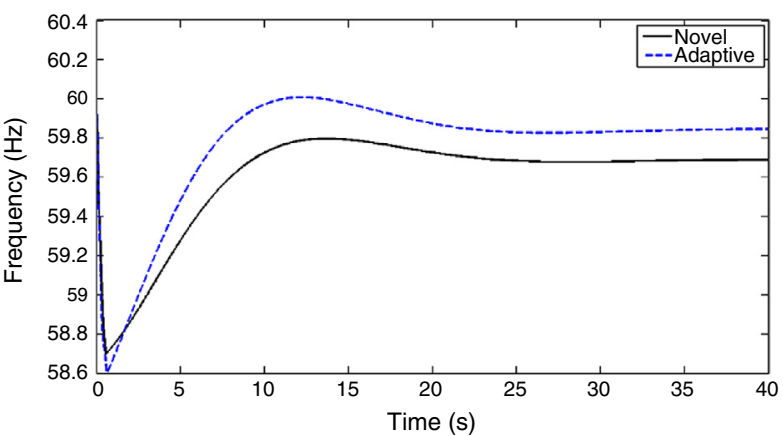

Fig. 8. Comparison of novel and adaptive load shedding schemes in case of $15 \%$ increase in $1 / H$.

a satisfactory performance when the inertia constant of the system decreases. The detailed results of this case study are given in Table 5. From the results presented in this table, it is clear that the proposed method not only results in a smaller amount of load shedding but also smaller frequency deviation.

However, when $1 / H$ used in the adaptive load shedding scheme is more than the actual value, the calculated power deficit will be less than actual amount of power deficit; therefore; the power deficit will not be fully compensated. Consequently, the frequency of system may decrease to the values lower than allowed limits.

In this case, the impact of decrease in $1 / H$ on the performance of the load shedding schemes is studied. Figure 9 shows that in case of a $10 \%$ decrease in $1 / H$, both of the load shedding methods can maintain the system frequency within the allowed limits. However, as it is shown in Figure 10, decreasing $1 / H$ by $15 \%$ the adaptive load shedding method is not successful in preventing the frequency from excursion lower than these limits. The detailed results of performance of both UFLS schemes in these two cases are given in Tables 6 and 7. The results presented in these tables show that by decreasing $1 / H$, the adaptive method does not shed enough load to maintain the frequency within allowed limits. However, the novel method shows an acceptable performance in this case. 
Table 5

Detailed result of novel and adaptive load shedding when the $1 / H$ is increased by $15 \%$.

\begin{tabular}{lllllll}
\hline Method & Step 1 & Step 2 & Step 3 & Step 4 & Total shed & $f_{\min }$ \\
\hline Novel & 0.1785 & 0.1506 & 0.2139 & 0.2214 & 0.7644 & 58.70 \\
Adaptive & 0.322 & 0.276 & 0.184 & 0 & 0.782 & 59.68 \\
\hline
\end{tabular}

Table 6

Detailed result of novel and adaptive load shedding when the $1 / H$ is decreased by $10 \%$.

\begin{tabular}{lllllll}
\hline Method & Step 1 & Step 2 & Step 3 & Step 4 & Total shed & $f_{\text {min }}$ \\
\hline Novel & 0.1596 & 0.123 & 0.2119 & 0.248 & 0.7425 & 58.87 \\
Adaptive & 0.252 & 0.216 & 0.144 & 0.108 & 0.72 & 59.49 \\
\hline
\end{tabular}

Table 7

Detailed result of novel and adaptive load shedding when the $1 / H$ is decreased by $15 \%$.

\begin{tabular}{lllllll}
\hline Method & Step 1 & Step 2 & Step 3 & Step 4 & Total shed & $f_{\text {min }}$ \\
\hline Novel & 0.1558 & 0.1391 & 0.1712 & 0.2826 & 0.7487 & 58.94 \\
Adaptive & 0.238 & 0.204 & 0.136 & 0.102 & 0.680 & 59.55 \\
\hline
\end{tabular}

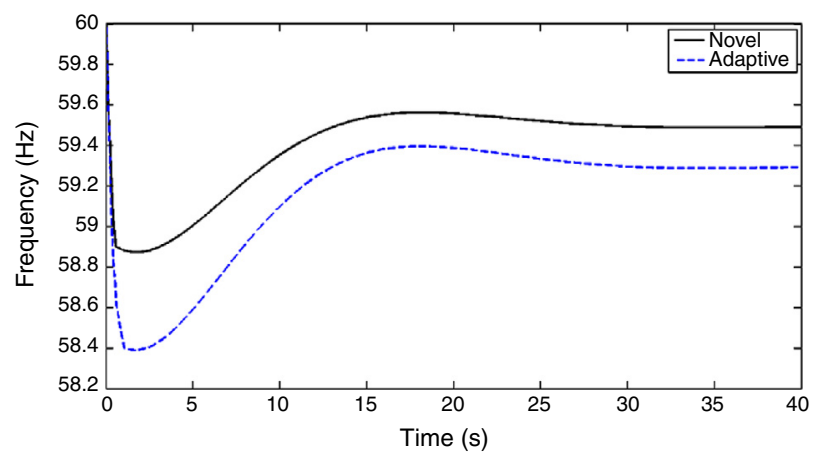

Fig. 9. Comparison of novel and adaptive load shedding schemes in case of $10 \%$ decrease in $1 / H$

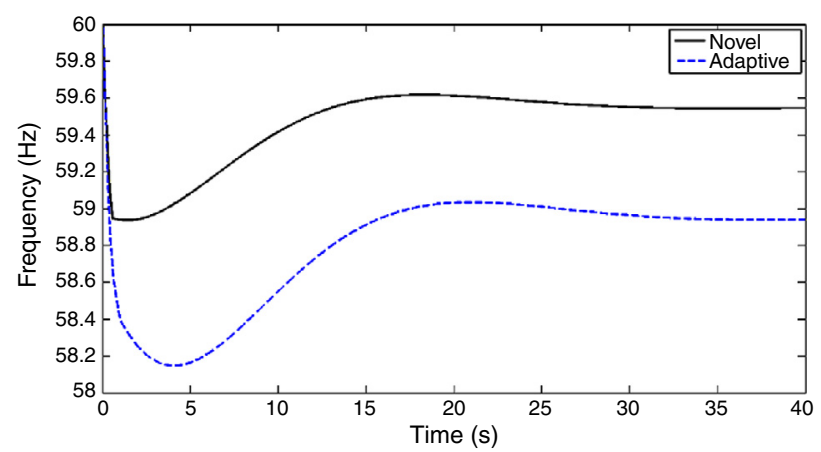

Fig. 10. Comparison of novel and adaptive load shedding schemes in case of $15 \%$ decrease in $1 / H$

This case study shows that the novel load shedding method is not sensitive to changes in inertia constant of power system and has an acceptable performance in this case.

\subsection{Case 3}

It is probable that generator outage occurs during the load shedding process as a result of a fault or malfunction of gen- erators underfrequency relays. This will result in an additional power deficit during the load shedding process. So, for maintaining the stability of power system, it is necessary for the load shedding schemes to consider this additional power deficit in the next steps of load shedding.

In this case study, the performance of both of the load shedding schemes in case of an additional 0.2 P.U. power deficit, $0.3 \mathrm{~s}$ after the initial 0.8 P.U. power deficit, is investigated. The adaptive load shedding method calculates the power deficit just after occurrence of the initial power deficit; so, it is not able to recognize the additional power deficit. Consequently, the additional power deficit is not compensated by the adaptive UFLS method and as Figure 11 shows, this results in excursion of frequency out of the permissible limits. But, the novel scheme, estimates the power deficit after every load shedding step. Therefore, it is able to recognize the additional power deficit and consider it in the next load shedding steps. Figure 11 certifies the proper performance of the novel UFLS scheme in case of additional power deficit during the load shedding process.

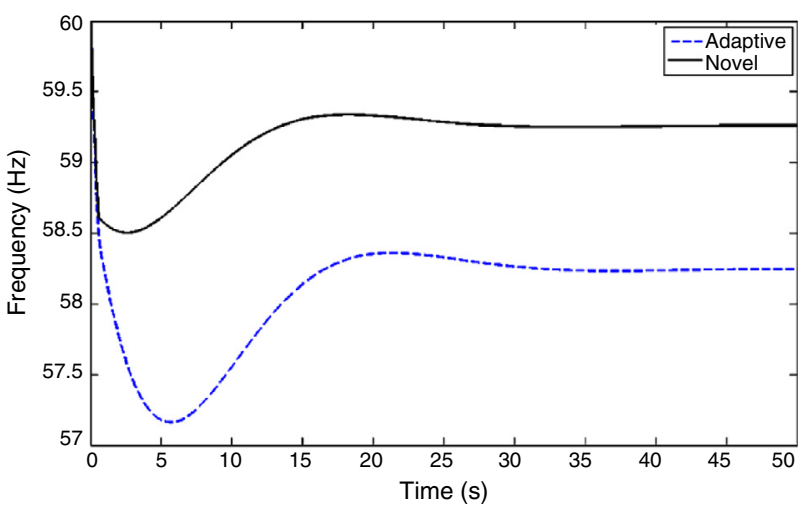

Fig. 11. Comparison of novel and adaptive load shedding schemes in case of occurrence of additional power deficit during the load shedding process. 


\section{Conclusion}

A novel load shedding method, based on forecasting the minimum frequency, is presented in this paper. It is shown that by using the proposed method, the minimum frequency is estimated with a high precision. Therefore, the power deficit can be estimated precisely; then, the proper value of load can be shed at the proper time. Based on simulation studies, the proposed UFLS scheme has been compared with one of newly proposed methods. Superiority of the proposed method in different case studies has been confirmed. It has been demonstrated that, in cases where parameters of system are not changed, the proposed method sheds less load and also results in a smaller frequency deviation. In addition, simulation results verify that the proposed load shedding scheme is independent of power system parameters and even in case of system parameter variation, it is able to keep the frequency within the allowed limits. Furthermore, it is proved that this method has a proper performance when an additional power deficit occurs due to outage of generating units during the load shedding process.

\section{Conflict of interest}

The authors have no conflicts of interest to declare.

\section{References}

Aik, D. L. H. (2006). A general-order system frequency response model incorporating load shedding: Analytic modeling and applications. IEEE Transactions on Power Systems, 21(2), 709-717.

Amjady, N., \& Fallahi, F. (2010). Determination of frequency stability border of power system to set the thresholds of under frequency load shedding relays. Energy Conversion and Management, 51(10), 1864-1872.

Anderson, P. M., \& Mirheydar, M. (1990). A low-order system frequency response model. IEEE Transactions on Power Systems, 5(3), 720-729.

Anderson, P. M., \& Mirheydar, M. (1992). An adaptive method for setting underfrequency load shedding relays. IEEE Transactions on Power Systems, 7(2), 647-655.

Berdy, J. (1968). Load shedding —An application guide. Schenectady, NY: General Electric Company Electric Utility Engineering Operation.

Chang-Chien, L. R., An, L. N., Lin, T. W., \& Lee, W. J. (2012). Incorporating demand response with spinning reserve to realize an adaptive frequency restoration plan for system contingencies. IEEE Transactions on Smart Grid, $3(3), 1145-1153$.

Chuvychin, V. N., Gurov, N. S., Venkata, S. S., \& Brown, R. E. (1996). An adaptive approach to load shedding and spinning reserve control during underfrequency conditions. IEEE Transactions on Power Systems, 11(4), $1805-1810$.

Concordia, C., Fink, L. H., \& Poullikkas, G. (1995). Load shedding on an isolated system. IEEE Transactions on Power Systems, 10(3), 1467-1472.

Delfino, B., Massucco, S., Morini, A., Scalera, P., \& Silvestro, F. (2001). Implementation and comparison of different under frequency load-shedding schemes. IEEE Power Engineering Society Summer Meeting, 2001, 1, 307-312.

Eberhart, R., \& Kennedy, J. (1995). A new optimizer using particle swarm theory. In IEEE proceedings of the sixth international symposium on micro machine and human science (pp. 39-43).

Hajiakbari Fini, M., Yousefi, G. R., \& Alhelou, H. H. (2016). Comparative study on the performance of many-objective and single-objective optimisation algorithms in tuning load frequency controllers of multiarea power systems. IET Generation, Transmission \& Distribution, 10(12), 2915-2923.

Halevi, Y., \& Kottick, D. (1993). Optimization of load shedding system. IEEE Transactions on Energy Conversion, 8(2), 207-213.

Hong, Y. Y., \& Chen, P. H. (2012). Genetic-based underfrequency load shedding in a stand-alone power system considering fuzzy loads. IEEE Transactions on Power Delivery, 27(1), 87-95.

Hong, Y. Y., \& Wei, S. F. (2010). Multiobjective underfrequency load shedding in an autonomous system using hierarchical genetic algorithms. IEEE Transactions on Power Delivery, 25(3), 1355-1362.

Ketabi, A., \& Hajiakbari Fini, M. (2014). An underfrequency load shedding scheme for islanded microgrids. International Journal of Electrical Power \& Energy Systems, 62, 599-660.

Ketabi, A., \& Hajiakbari Fini, M. (2015a). An adaptive set-point modulation technique to enhance the performance of load frequency controllers in a multi-area power system. Journal of Electrical Systems and Information Technology, 2(3), 391-405.

Ketabi, A., \& Hajiakbari Fini, M. (2015b). An underfrequency load shedding scheme for hybrid and multiarea power systems. IEEE Transactions on Smart Grid, 6(1), 82-91.

Kundur, P. (1994). . N. J. Balu, \& M. G. Lauby (Eds.), Power system stability and control (Vol. 7) New York: McGraw-Hill.

Laghari, J. A., Mokhlis, H., Bakar, A. H. A., \& Mohamad, H. (2013). Application of computational intelligence techniques for load shedding in power systems: A review. Energy Conversion and Management, 75, 130-140.

Larsson, M. (2005). An adaptive predictive approach to emergency frequency control in electric power systems. In Proceedings of the 44th IEEE conference on decision and control (pp. 4434-4439).

Lokay, H. E., \& Burtnyk, V. (1968). Application of underfrequency relays for automatic load shedding. IEEE Transactions on Power Apparatus and Systems, 3, 776-783.

Lopes, J. A. P., \& Mitchell, M. A. (2000). Optimum determination of underfrequency load shedding strategies using a genetic algorithm approach. In Proceedings of the Thirty-Second Annual North American Power Symposium.

Maliszewski, R. M., Dunlop, R. D., \& Wilson, G. L. (1971). Frequency actuated load shedding and restoration. Part I-Philosophy. IEEE Transactions on Power Apparatus and Systems, 4, 1452-1459.

Pahwa, S., Scoglio, C., Das, S., \& Schulz, N. (2013). Load-shedding strategies for preventing cascading failures in power grid. Electric Power Components and Systems, 41(9), 879-895.

Rudez, U., \& Mihalic, R. (2011a). Analysis of underfrequency load shedding using a frequency gradient. IEEE Transactions on Power Delivery, 26(2), $565-575$

Rudez, U., \& Mihalic, R. (2011b). Monitoring the first frequency derivative to improve adaptive underfrequency load-shedding schemes. IEEE Transactions on Power Systems, 26(2), 839-846.

Rudez, U., \& Mihalic, R. (2011c). A novel approach to underfrequency load shedding. Electric Power Systems Research, 81(2), 636-643.

Rudez, U., \& Mihalic, R. (2015). Predictive underfrequency load shedding scheme for islanded power systems with renewable generation. Electric Power Systems Research, 126, 21-28.

Sigrist, L., Egido, I., Sanchez-Ubeda, E. F., \& Rouco, L. (2010). Representative operating and contingency scenarios for the design of UFLS schemes. IEEE Transactions on Power Systems, 25(2), 906-913.

Terzija, V. V., \& Koglin, H. J. (2002). Adaptive underfrequency load shedding integrated with a frequency estimation numerical algorithm. IEE Proceedings - Generation, Transmission and Distribution, 149(6), 713-718.

Thalassinakis, E. J., \& Dialynas, E. N. (2004). A Monte-Carlo simulation method for setting the underfrequency load shedding relays and selecting the spinning reserve policy in autonomous power systems. IEEE Transactions on Power Systems, 19(4), 2044-2052.

Van Den Bergh, F., \& Engelbrecht, A. P. (2001). Training product unit networks using cooperative particle swarm optimisers. IEEE Proceedings - International Joint Conference on Neural Networks, 1, 126-131. 